\title{
Densità di energia di deformazione locale e resistenza a fatica di giunti saldati di geometria complessa
}

\author{
M. Zappalorto, F. Berto, P. Lazzarin \\ Università di Padova, Dipartimento di Tecnica e Gestione dei sistemi industriali, Stradella San Nicola 3, \\ 36100 Vicenza \\ e-mail: zappalorto@gest.unipd.it,plazzarin@gest.unipd.it
}

RIASSUNTO. Un recente criterio basato sul valore medio della densità di energia di deformazione (SED) in un volume di controllo è applicato a diverse serie di dati sperimentali tratti dalla letteratura, relativi a giunti saldati di geometria complessa realizzati in acciaio. Il volume di controllo è rappresentato da un settore circolare di raggio pari a $0.28 \mathrm{~mm}$, centrato sul piede o sulla radice dei cordoni di saldatura. Entrambe le regioni sono modellate come intagli $\mathrm{V}$ non raccordati con differenti angoli di apertura.

La densità di energia di deformazione viene valutata direttamente da modelli agli elementi finiti tridimensionali. I dati sperimentali, riconvertiti in termini energetici, si posizionano all'interno di una banda di dispersione recentemente proposta in letteratura. La banda sintetizzava più di 650 dati sperimentali relativi a giunti saldati con cordone d'angolo, con rotture innescate indifferentemente al piede o alla radice dei cordoni di saldatura.

ABSTRACT. A recent criterion based on the local strain energy density (SED) averaged over a given control volume is applied to well-documented experimental data taken from the literature, all related to steel welded joints of complex geometry. This small size volume embraces the weld root or the weld toe, both regions being modelled as sharp (zero notch radius) V-notches with different opening angles.

The SED is evaluated from three-dimensional finite element models by using a circular sector with a radius equal to $0.28 \mathrm{~mm}$. The data expressed in terms of the local energy fall in a scatter band recently reported in the literature, based on about 650 experimental data related to fillet welded joints made of structural steel with failures occurring at the weld toe or at the weld root.

PAROLE CHIAVE. Notch Stress Intensity Factor, energia di deformazione, giunti saldati, resistenza a fatica

\section{INTRODUZIONE}

Nella letteratura recente è stato ampiamente dimostrato come la resistenza a fatica di giunzioni saldate ad arco in acciaio da costruzione o in lega leggera possa essere sintetizzata in termini di fattori di intensificazione delle tensioni o "Notch Stress Intensity Factors" (N-SIFs) [1-3]. Il problema della variabilità dei raggi di raccordo al piede e alla radice dei cordoni di saldatura viene superato nell'approccio N-SIF modellando i cordoni come intagli acuti a $\mathrm{V}$; le distribuzioni di tensione in corrispondenza dei punti critici presentano quindi un carattere asintotico e gli N-SIF quantificano proprio la loro intensità. Poiché le distribuzioni sono sensibili alle dimensioni assolute del giunto, l'effetto scala è interamente conglobato nel valore dell'N-SIF [1-3].

Quando l'angolo di apertura $2 \alpha$ dell'intaglio è abbastanza grande da rendere il modo II non singolare $\left(2 \alpha>102^{\circ}\right.$, condizione generalmente soddisfatta in tutti i giunti con cordoni d'angolo), il comportamento a fatica in presenza di sollecitazioni di trazione o flessione dipende solo dal fattore di intensificazione di modo $\mathrm{I}, \Delta \mathrm{K}_{1}$ [1-3]. Tale parametro, che era naturale pensare idoneo a controllare la fase di innesco delle cricche di fatica, è invece risultato efficace anche nell'operare una sintesi della vita finale dei giunti saldati; ciò accade perché nei campioni testati in laboratorio la maggior parte della vita a fatica è spesa come innesco e propagazione di cricche corte, localizzate all'interno della zona di singolarità governata dai campi asintotici iniziali [3].

Gli N-SIF permettono di superare il complesso problema legato alla propagazione di cricche multiple e della loro possibile interazione su piani diversi, fenomeni variabili in funzione del tipo di sollecitazione e delle irregolarità presenti al piede dei cordoni. La Fig. 1 riassume numerosi dati sperimentali in funzione del fattore di intensificazione delle tensioni di modo I. La sintesi riguarda giunti a croce e a $\mathrm{T}$, soggetti a trazione o a flessione e caratterizzati da uno spessore dei piatti principali variabile tra $3 \mathrm{e}$ $100 \mathrm{~mm}$. La variabilità dei piatti traversali risulta ancora più pronunciata, con uno spessore variabile tra 3 e 220 $\mathrm{mm}$. Le rotture si manifestavano sempre al piede dei cordoni di saldatura, in presenza di un angolo apertura di $135^{\circ}$ tra piatti principali e superficie inclinata dei cordoni.

Dal punto di vista teorico, la banda di dispersione riportata in Fig. 1 non può essere estesa a giunzioni saldate che manifestano rotture alla radice dei cordoni, né a giunzioni 


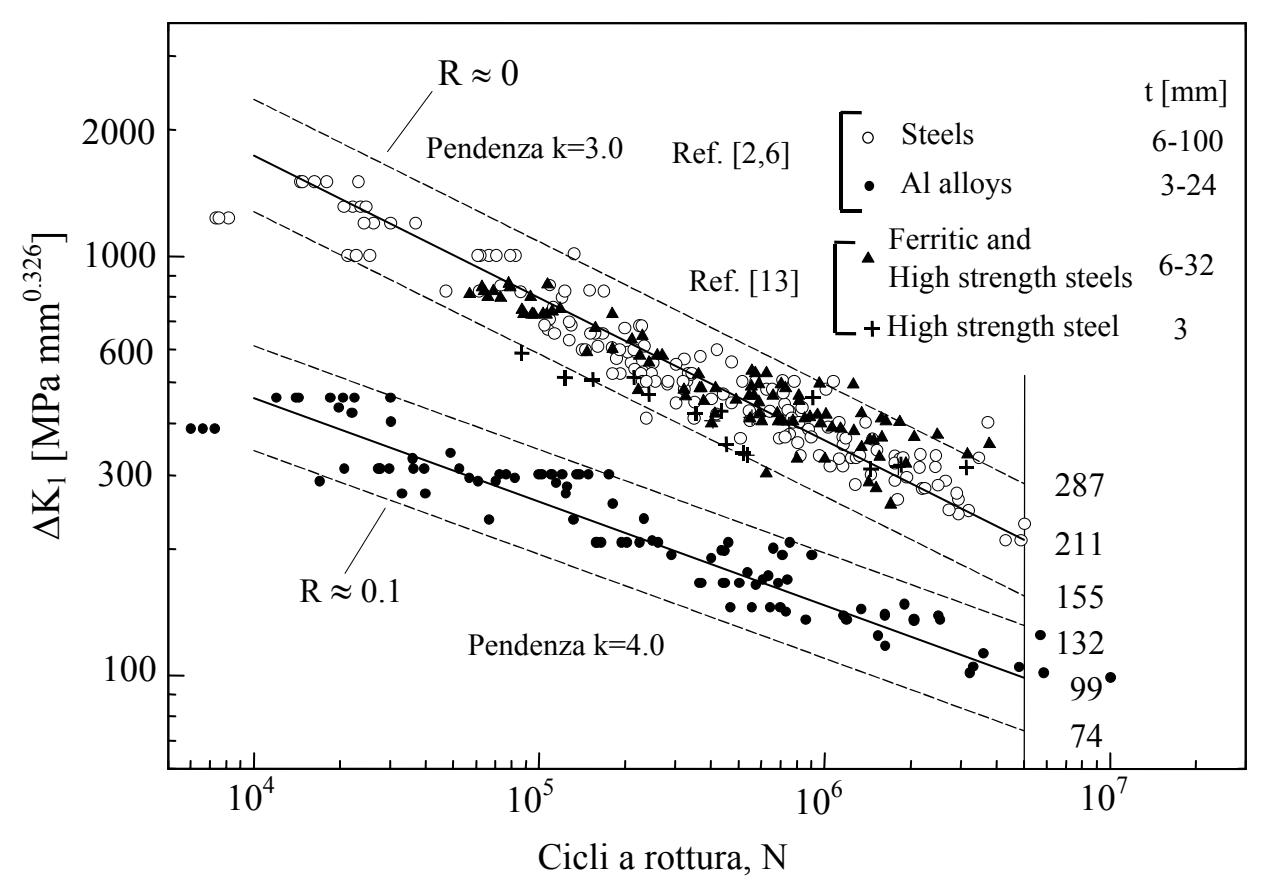

Figura 1. Resistenza a fatica di giunti saldati in acciaio e lega leggera in funzione del fattore di intensificazione delle tensioni di modo I. Geometrie a croce e a T, con cordone portante. Angolo di apertura di $135^{\circ}$; $\mathrm{t}$ spessore dei piatti principali; banda di dispersione relativa a valori medi \pm 2 deviazioni standard $\left(\mathrm{P}_{\mathrm{S}}=2.3-97.7 \%\right)$. La Figura è tratta dal riferimento [7].

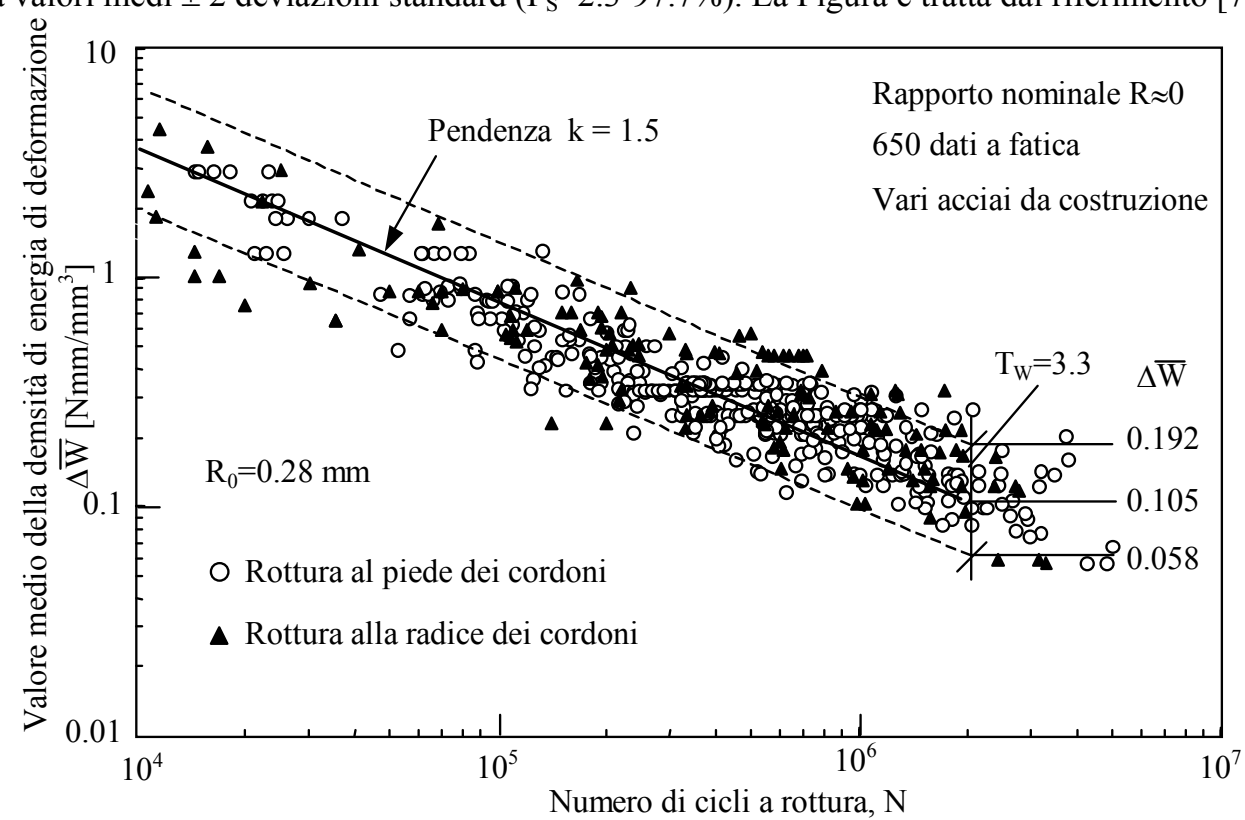

Figura 2. Resistenza a fatica in funzione del valore medio della densità di energia di deformazione presente in un settore semicircolare di raggio $R_{\mathrm{C}}$ centrato sull'apice dell'intaglio a $\mathrm{V}$ [7-8]. Nel caso di giunti saldati realizzati con acciai da costruzione il raggio critico $R_{\mathrm{C}}$ vale $0.28 \mathrm{~mm}$.

con angoli di apertura al piede sensibilmente diversi da $135^{\circ}$. Questo è dovuto al fatto che le unità di misura degli N-SIF cambiano proprio in funzione dell'angolo di apertura. Il problema è stato superato in recenti lavori [4-9] usando come parametro rappresentativo della resistenza a fatica il valore medio della densità di energia di deformazione $\Delta \mathrm{W}$ calcolata in un volume finito centrato sul piede o sulla radice dei cordoni. In linea di principio tale energia è funzione dei fattori di intensificazione di modo I e di modo II nei casi piani [4], di Modo I, II e III nel caso di sollecitazioni multiassiali $[6,9]$.

Una banda in termini di $\Delta \mathrm{W}$ è stata inizialmente proposta nelle referenze $[4,5]$ sulla base di circa 300 dati sperimentali relativi ad acciai da costruzione saldati ed è stata successivamente verificata utilizzando altri 400 dati che si sono resi disponibili nel tempo [7].

La stessa banda è risultata valida non solo per i giunti con cordoni d'angolo ma anche per i giunti saldati testa a testa [8]. Una sintesi parziale è presentata in Fig. 2 relativamente a giunti realizzati con acciai da costruzione, interessati da rotture al piede e alla radice dei cordoni di saldatura [7-8].

L'indice $T_{\mathrm{w}}$ che fornisce la larghezza di banda riferita a valori medi $+/$ - due deviazioni standard vale 3.3. Passando dal range di densità di energia di deformazione a un range di tensione locale equivalente e ai livelli di probabilità di sopravvivenza del 10-90\%, l'indice scende a $T_{\sigma}=$ 


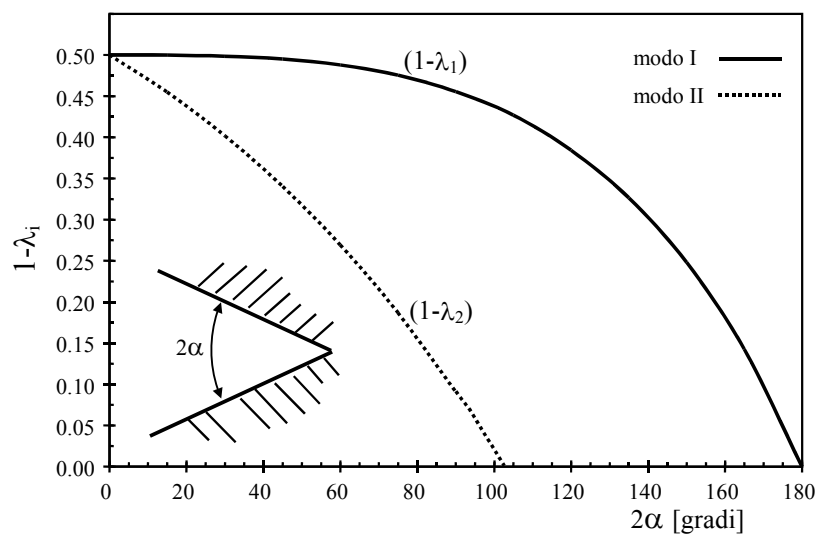

Figura 3. Grado di singolarità $1-\lambda$ dei campi di tensione in prossimità di intagli a $\mathrm{V}$ non raccordati in funzione dell'angolo di apertura.

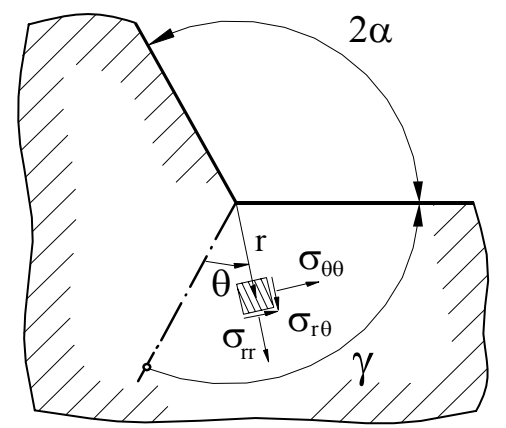

Figura 4. Sistema di coordinate polari centrato sull'apice dell'intaglio.

\begin{tabular}{cccccc}
\hline $2 \alpha / \pi$ & $\gamma / \pi$ & $\lambda_{1}$ & $\lambda_{2}$ & $\mathrm{I}_{1}$ & $\mathrm{I}_{2}$ \\
\hline 0 & 1 & 0.5000 & 0.5000 & 0.8450 & 2.1450 \\
$3 / 4$ & $5 / 8$ & 0.6736 & 1.3021 & 0.6201 & 1.1505
\end{tabular}

Tabella 1. Valore dei parametri presenti nelle equazioni (1-3) ottenuti con l'ipotesi di Beltrami e con un coefficiente di Poisson $v=0.3$.

1.50 , valore che caratterizza la banda normalizzata S-N di Haibach [10].

L'obiettivo del presente lavoro è quello estendere l'uso del criterio dell'energia locale a un elevato numero di dati sperimentali tratti dalla letteratura [11-13] relativi a giunti saldati in acciaio con geometrie tridimensionali complesse e di confrontarli con la banda di dispersione già citata.

\section{PREMESSE ANALITICHE}

Il grado di singolarità $1-\lambda_{\mathrm{i}}$ dei campi di tensione in prossimità di intagli a $\mathrm{V}$ non raccordati varia in funzione dell'angolo di apertura (Fig. 3), riducendosi progressivamente rispetto al caso di una cricca dove il grado di singolarità è 0.5 per i modi di sollecitazione I e II $[1,14]$. L'intensità delle distribuzioni di tensione asintotiche presenti di fronte all'apice dell'intaglio a $\mathrm{V}$ non raccordato è generalmente espressa in funzione degli N-SIF.

In un sistema in coordinate polari $(r, \theta)$ avente l'origine centrata sull'apice (Fig. 4), le distribuzioni lineari elasti- che delle tensioni possono essere espresse in forma generale come:

$$
\begin{array}{ll}
\text { Modo I } & \sigma_{\mathrm{ij}}^{(1)}(\mathrm{r}, \theta)=\mathrm{K}_{1}^{\mathrm{N}} \cdot \widetilde{\sigma}_{\mathrm{ij}}^{(1)}(\theta) \cdot \mathrm{r}^{\lambda_{1}-1} \\
\text { Modo II } & \sigma_{\mathrm{ij}}^{(2)}(\mathrm{r}, \theta)=\mathrm{K}_{2}^{\mathrm{N}} \cdot \widetilde{\sigma}_{\mathrm{ij}}^{(2)}(\theta) \cdot \mathrm{r}^{\lambda_{2}-1}
\end{array}
$$

dove $\mathrm{K}_{1}^{\mathrm{N}}$ e $\mathrm{K}_{2}^{\mathrm{N}}$, sono gli N-SIF di modo I e II, $\widetilde{\sigma}_{\mathrm{ij}}(\theta \theta)$ sono le funzioni angolari e, infine, $\lambda_{1}$ e $\lambda_{2}$ gli autovalori del problema lineare elastico.

Vale per gli N-SIF la seguente definizione [15]:

$$
\begin{aligned}
& K_{1}^{N}=\sqrt{2 \pi} \lim _{r \rightarrow 0^{+}}\left[\sigma_{\theta \theta}(r, 0)\right] r^{1-\lambda_{1}} \\
& K_{2}^{N}=\sqrt{2 \pi} \lim _{r \rightarrow 0^{+}}\left[\sigma_{r \theta}(r, 0)\right] r^{1-\lambda_{2}}
\end{aligned}
$$

Nell'ipotesi di deformazione piana, l'energia di deformazione mediata su un settore circolare di raggio $R_{\mathrm{C}}$ che abbraccia l'apice dell'intaglio può essere espressa mediante la relazione [4-9]: 


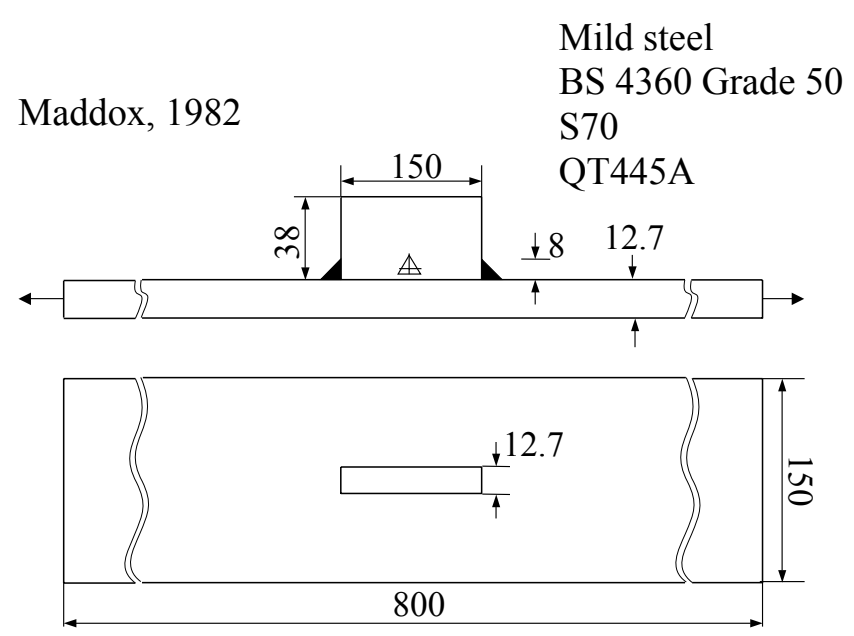

(a)

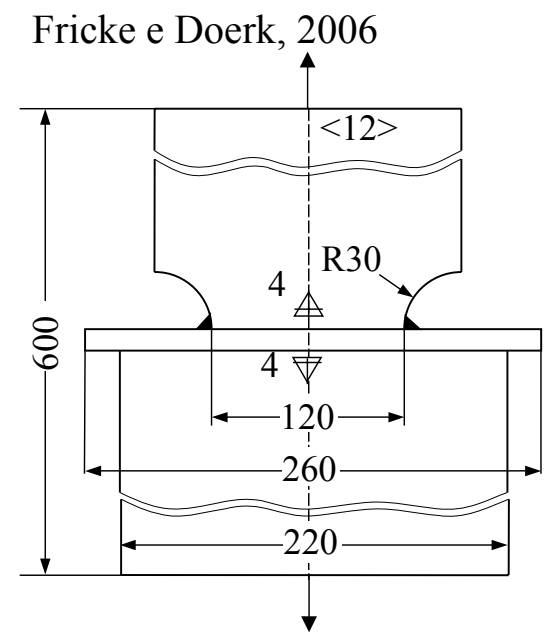

(c)
Lihavainen e Marquis, 2003

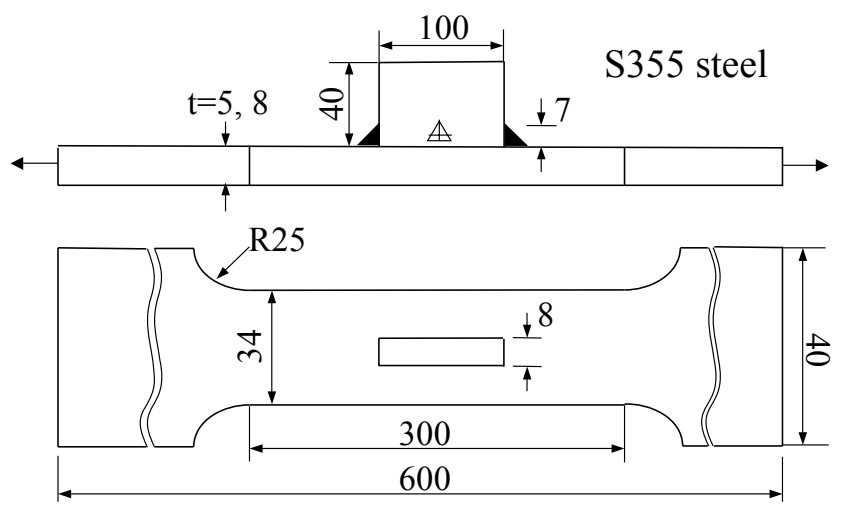

(b)

Fricke e Doerk, 2006

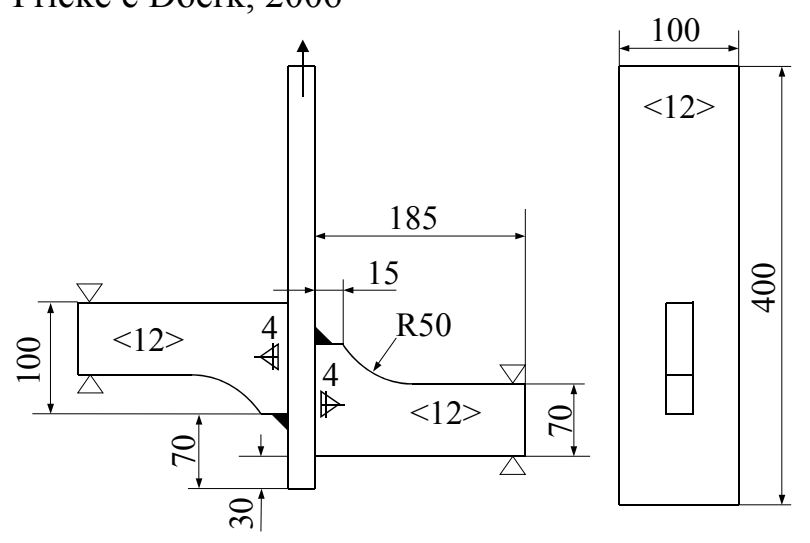

(d)

Figura 5. Geometrie dei giunti saldati analizzati. I dati originali sono relativi ai riferimenti [11-13]. Tutte le dimensioni sono in $\mathrm{mm}$.

$$
\Delta \overline{\mathrm{W}}=\frac{\mathrm{c}_{\mathrm{w}}}{\mathrm{E}}\left\{\frac{\mathrm{I}_{1}}{4 \lambda_{1} \gamma}\left[\frac{\Delta \mathrm{K}_{1}^{\mathrm{N}}}{\mathrm{R}_{\mathrm{C}}^{1-\lambda_{1}}}\right]^{2}+\frac{\mathrm{I}_{2}}{4 \lambda_{2} \gamma}\left[\frac{\Delta \mathrm{K}_{2}^{\mathrm{N}}}{\mathrm{R}_{\mathrm{C}}^{1-\lambda_{2}}}\right]^{2}\right\}
$$

dove il coefficiente $c_{\mathrm{w}}$ permette di tenere conto dell'influenza del rapporto nominale di ciclo $R$ nel solo caso di giunti soggetti a distensione post-saldatura.

Tutti gli altri parametri in gioco sono riportati in Tab. 1 con riferimento al criterio della densità di energia totale di deformazione (ipotesi di Beltrami) e a un coefficiente di Poisson $v=0.3$.

Il parametro $c_{\mathrm{w}}$, definito in condizioni lineari elastiche, può essere ottenuto per mezzo delle seguenti relazioni [6]:

$$
c_{w}(R)=\left\{\begin{array}{ccc}
\frac{1+R^{2}}{(1-R)^{2}} & \text { se } & -1 \leq R<0 \\
\frac{1-R^{2}}{(1-R)^{2}} & \text { se } & 0 \leq R \leq 1
\end{array}\right.
$$

Passando da $R=0$ a $R=-1, c_{\mathrm{w}}$ scende da 1.0 a 0.5 ; ad un dimezzamento della densità di energia locale corrisponde un aumento della resistenza a fatica in termini di tensione locale di un fattore $1 / .707=1.41$, simile al coefficiente empirico 1.33 suggerito dalla CNR UNI 10011. Ovviamente, se i giunti non subiscono alcun trattamento di distensione post-saldatura, la dipendenza dal rapporto nominale di ciclo $R$ scompare e il parametro $c_{\mathrm{w}}$ deve considerarsi unitario. E' noto infatti che nelle strutture reali soggetti ad elevati stati di tensioni residue la resistenza a fatica è sostanzialmente indipendente dal rapporto nominale di ciclo [16].

\section{RESISTENZA A FATICA DI GIUNTI} SALDATI DI GEOMETRIA COMPLESSA BASATA SULLA DENSITA' DI ENERGIA DI DEFORMAZIONE LOCALE

Sono stati riconsiderati alcuni dati sperimentali relativi a particolari strutturali saldati in acciaio tratti da precedenti lavori [11-13]. I principali parametri geometrici così come le condizioni di carico e di vincolo erano perfettamente definite nei lavori originali e sono rappresentati schematicamente in Fig. 5. 


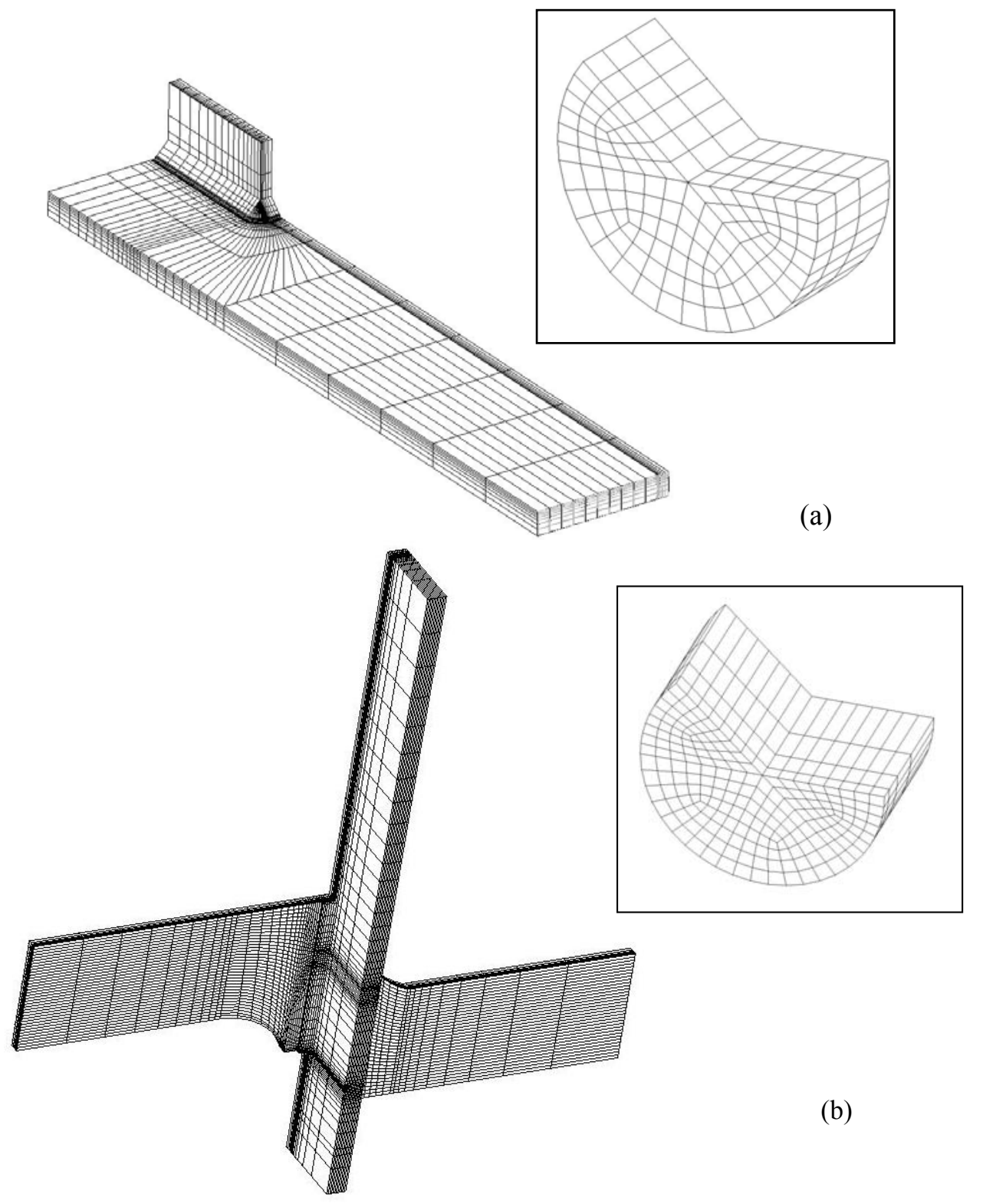

Figura 6. Esempio di modellazione del giunto ad irrigidimento longitudinale già analizzato da Maddox [11] (a) e del giunto cruciforme già analizzato da Fricke e Doerk [13] (b); modelli complessivi e particolari relativi alla modellazione del volume strutturale.

\subsection{Dati di Maddox.}

La prima serie rianalizzata riguarda i dati di resistenza a fatica già discussi da Maddox [11]. I componenti utilizzati nelle prove sperimentali consistono in giunti con un irrigidimento longitudinale saldato su un solo lato del piatto principale, avente uno spessore di $12.7 \mathrm{~mm}$ (Fig. 5a) e realizzati in quattro differenti tipologie di acciai (i.e. Mild steel, BS 4360 Grade 50, S70 and QT445A). I componenti sono stati testati a trazione in condizioni as-welded utilizzando cinque differenti rapporti nominali di carico: $R=-\infty,-1,0,0.5$ e 0.67 .

Tutti i componenti sono stati portati a completa rottura, fatta eccezione per il caso $R=-\infty$ in cui la velocità di propagazione manifestava un decremento al propagare delle cricche di fatica; per questa serie $i$ test erano stati arrestati prima del completo cedimento. In tutti i casi considerati la nucleazione delle cricche di fatica avveniva in corrispondenza del piede del cordone.

\subsection{Dati di Lihavainen e Marquis.}

I giunti ad irrigidimento longitudinale non portante già analizzati da Lihavainen e Marquis [12] sono rappresentati in Fig. 5b. I giunti erano realizzati in acciaio S355 J0 utilizzando piatti principali di spessore $5 \mathrm{~mm}$ e $8 \mathrm{~mm}$. Le prove di fatica erano state condotte a trazione con un rapporto di ciclo pari a $R=0.1$. Per tutti i componenti considerati la nucleazione delle cricche di fatica avveniva in corrispondenza del piede del cordone.

\subsection{Dati di Fricke e Doerk.}

L'ultima serie di dati sperimentali riguarda i giunti saldati sottoposti a sperimentazione da Fricke e Doerk [13] e rappresentati in Fig. 5c-d. I dati di resistenza a fatica erano stati ottenuti con prove di trazione (Fig. 5c) e di flessione a tre punti (Fig. 5d). I dettagli saldati erano stati testati sia in condizioni as-welded (con due differenti rapporti nominali di carico, $R=0$ and $R=0.5$ ), che stressrelieved ( $\operatorname{con} R=0$ ). 


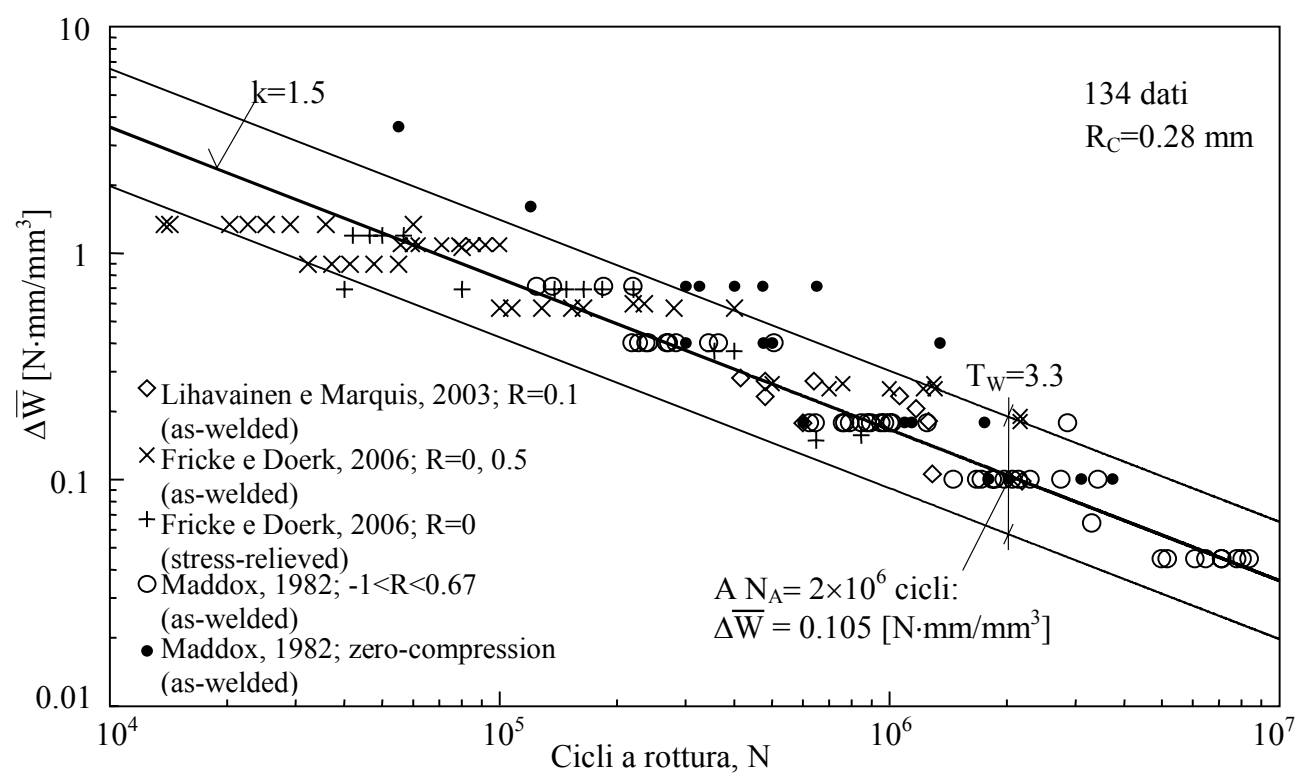

Figura 7. Resistenza a fatica di giunti saldati in acciaio strutturale di geometria complessa in termini di densità di energia locale; i dati originali sono riportati nei riferimenti [11-13]. Confronto con la banda di dispersione proposta da Lazzarin et al. [5] $\left(\mathrm{P}_{\mathrm{S}}=2.3-97.7\right)$.

I componenti di Fig. 5c testati in condizioni as-welded esibivano un comportamento a rottura anomalo; in molti casi infatti le cricche di fatica innescavano alla radice del cordone di saldatura, in corrispondenza della zona centrale del piatto principale, nonostante lo stato tensionale non fosse massimo in quel punto.

Nei componenti stress-relieved invece, la nucleazione avveniva sia al piede che alla radice del cordone di saldatura. Infine, la maggior parte dei componenti rappresentati in Fig. 5d manifestava prevalentemente una nucleazione alla radice.

Per riconvertire i dati sperimentali in termini energetici la densità di energia di deformazione è sempre stata valutata su un volume di controllo che abbraccia le zone di innesco delle cricche di fatica indicate dagli autori nei lavori originali.

La densità di energia di deformazione è stata ottenuta mediante analisi tridimensionali agli elementi finiti eseguite con il software Ansys $9.0^{\circledR}$, modellando il volume di controllo come un settore circolare tridimensionale di raggio $R_{\mathrm{C}}=0.28 \mathrm{~mm}$, posizionato al piede o alla radice del cordone di saldatura (Fig. 6).

Poiché i dati fanno riferimento sia a giunti sottoposti a trattamento di distensione delle tensioni residue (stressrelieved) con $R=0$, sia a giunti testati allo stato as-welded con differenti rapporti nominali di carico $(-\infty<R<0.67)$, nella sintesi il coefficiente correttivo $c_{\mathrm{W}}$ è sempre stato posto pari al valore unitario.

Nella Fig. 7 i dati sperimentali riconvertiti in termini energetici sono confrontati con una banda di dispersione già riportata nel riferimento [5]; 1'accordo è molto soddisfacente, tranne per alcuni dati relativi alle serie già analizzate da Maddox [11] e caratterizzati da un rapporto di ciclo pari a $R=-\infty$ (pura compressione).

L'indice di dispersione $T_{\mathrm{W}}$ relativo alle probabilità di sopravvivenza $P_{\mathrm{S}}=2.3 \%$ e $97.7 \%$ è risultato pari a 3.3 e se riconvertito in termini di tensione locale equivalente e probabilità di sopravvivenza $P_{\mathrm{S}}=10 \%$ e $90 \%$ risulta 1.5 , in perfetto accordo con la banda normalizzata suggerita da Haibach [10].

\section{CONCLUSIONI}

Nel presente lavoro sono state considerate diverse serie di dati di resistenza a fatica tratti dalla letteratura e relativi a unioni saldate in acciaio di geometria complessa. Per tali serie risultavano precisati tutti i parametri che consentono di descrivere con precisione la geometria locale in corrispondenza del piede e della radice dei cordoni di saldatura. I dati sperimentati sono stati rianalizzati utilizzando un criterio locale basato sul valore medio della densità di energia di deformazione, criterio già messo a punto e applicato a giunti di geometria diversa, soggetti a trazione o flessione. Il metodo energetico consente di sintetizzare dati di resistenza a fatica indipendentemente dal punto di innesco delle cricche e dall'angolo di apertura al piede dei cordoni.

I dati di resistenza a fatica, riconvertiti in termini di densità di energia locale, sono stati confrontati con una banda di sintesi recentemente proposta in letteratura. L'accordo con la banda di sintesi è risultato soddisfacente. L'indice di dispersione, se riconvertito in termini di tensione media locale, piuttosto che in termini di densità media di energia locale, appare perfettamente in linea con le indicazioni fornite dalla banda normalizzata di Haibach.

\section{BIBLIOGRAFIA}

[1] P. Lazzarin, R. Tovo, A notch stress intensity approach to the stress analysis of welds. Faigue and Frac- 
ture of Engineering Materials and Structures, 21 (1998) 1089-1103.

[2] B. Atzori, P. Lazzarin, R. Tovo, From the local stress approach to fracture mechanics: a comprehensive evaluation of the fatigue strength of welded joints. Fatigue and Fracture of Engineering Materials and Structures, 22 (1999) 369-382.

[3] P. Lazzarin, P. Livieri, Notch Stress Intensity Factors and fatigue strength of aluminium and steel welded joints. International Journal of Fatigue, 23 (2001) 225232.

[4] P. Lazzarin, R. Zambardi, A finite-volume-energy based approach to predict the static and fatigue behaviour of components with sharp V-shaped notches. International Journal of Fracture, 12 (2001) 275-298.

[5] P. Lazzarin, T. Lassen, P. Livieri. A Notch Stress Intensity approach applied to fatigue life predictions of welded joints with different local toe geometry. Fatigue and Fracture of Engineering Materials and Structures, 26 (2001) 49-58.

[6] P. Lazzarin, C.M. Sonsino, R. Zambardi. A Notch Stress Intensity approach to predict the fatigue behaviour of $\mathrm{T}$ butt welds between tube and flange when subjected to in-phase bending and torsion loading. Fatigue and Fracture of Engineering Materials and Structures, 27 (2004) 127-141.

[7] Livieri P, Lazzarin P. Fatigue strength of steel and aluminium welded joints based on generalised stress intensity factors and local strain energy values. International Journal of Fracture, 133 (2005) 247-276.
[8] Lazzarin P, Berto F, Radaj D. Uniform fatigue strength of butt and fillet welded joints in terms of the local strain energy density. Proc. Fatigue 2006, Atlanta, USA.

[9] P. Lazzarin, P. Livieri, F. Berto, M. Zappalorto. Engineering Fracture Mechanics. 2007; in press (available on line).

[10] E. Haibach. Service fatigue strength - methods and data for structural analysis. Springer Verlag, Berlin, 2002.

[11] S.J. Maddox. Influence of tensile residual stresses on the fatigue behavior of welded joints in steel. ASTM STP.,776 (1982) 63-96.

[12] V.M. Lihavainen, G. Marquis. Fatigue strength of a longitudinal attachment improved by ultrasonic impact treatment. IIW. 2003; Document XIII-1990-03.

[13] W. Fricke, O. Doerk. Simplified approach to fatigue strength assessment of fillet-welded attachment ends. International Journal of Fatigue, 28 (2006) 141-150.

[14] M.L. Williams. Stress singularities resulting from various boundary conditions in angular corners of plates in extension. ASME Journal of Applied Mechanics, 19 (1952) 526-528

[15] R. Gross, A. Mendelson. Plane elastostatic analysis of $\mathrm{V}$-notched plates. International Journal of Fracture Mechanics, 8 (1972) 267-327.

[16] B. Atzori. Trattamenti termici e resistenza a fatica delle strutture saldate. Rivista Italiana della Saldatura, 1 (1983) 3-16. 